\title{
When does rotation disrupt letter encoding? Testing the resilience of letter detectors in the initial moments of processing
}

\author{
Manuel Perea $^{1,2} \cdot$ Marta Vergara-Martínez $^{3} \cdot$ Ana Marcet $^{1} \cdot$ Reem Abu Mallouh ${ }^{1} \cdot$ María Fernández-López $^{1}$ \\ Published online: 27 January 2020 \\ (C) The Psychonomic Society, Inc. 2020
}

\begin{abstract}
Previous research has reported that both letter and word identification are slower when the stimuli are presented at rotations above $45^{\circ}$ than when presented in their canonical horizontal view. Indeed, influential models of word recognition posit that letter detectors in the visual word recognition system are disrupted by rotation angles above $40^{\circ}$ or $45^{\circ}$ (e.g., Local Combinations Detector model; Dehaene, Cohen, Sigman, \& Vinckier, 2005, Trends in Cognitive Sciences). However, recent experiments have shown robust masked identity/form priming effects for $90^{\circ}$ rotated words, thus calling into question this assumption. Here we aimed to isolate the degree to which letter detectors are disrupted when manipulating letter rotation in three masked identity priming letter match experiments. Probes and targets were always presented in the canonical upright position, whereas forwardly masked primes were rotated in different angles. The rotation angles were $0^{\circ}$ versus $45^{\circ}$ (Experiment 1 ), $22.5^{\circ}$ versus $67.5^{\circ}$ (Experiment 2), and $45^{\circ}$ versus $90^{\circ}$ (Experiment 3). Results showed a sizeable masked identity priming effect regardless of the rotation angle, hence demonstrating that letter detectors are not disrupted by rotations smaller than $90^{\circ}$ in the early moments of letter processing. This pattern suggests that letter detectors are more resilient to changes in visual form than predicted by the LCD model.
\end{abstract}

Keywords Masked priming $\cdot$ Rotation $\cdot$ Word recognition $\cdot$ Letter recognition

\section{Introduction}

The processes underlying visual word identification are tolerant to noise and variations in shape of the words' constituent letters (see Grainger, 2018; Grainger \& Dufau, 2012, for reviews). One of the most striking demonstrations was provided by Hannagan, Ktori, Chanceaux, and Grainger (2012) with briefly presented text-based CAPTCHAs (Completely Automated Public Turing test to tell Computers and Humans Apart; e.g., Unaque). They conducted a lexical decision experiment ("does the stimulus form a word?") in which an uppercase printed target was preceded by a forwardly masked lowercase CAPTCHA prime for $50 \mathrm{~ms}$. The prime was nominally

Manuel Perea

mperea@uv.es

1 ERI-Lectura and Department of Methodology, Universitat de València, Av. Blasco Ibáñez, 21, 46010 Valencia, Spain

2 Universidad Nebrija, Madrid, Spain

3 ERI-Lectura and Department of Educational and Developmental Psychology, Universitat de València, Valencia, Spain identical or unrelated to the target. Results showed substantially faster responses in the identity than in the unrelated condition. Hannagan et al. (2012) concluded that the word recognition system is tolerant to "global continuous input transforms and small letter rotations" (p. 2).

Clearly, the presence of masked identity priming effects with CAPTCHAs or other degraded stimuli (e.g., handwritten words; see Perea, Marcet, Uixera, \& Vergara-Martínez, $2018 \mathrm{~b}$, for review) is quite impressive. However, it is difficult to pinpoint the locus of this effect because the letters in degraded stimuli are distorted across several dimensions. To avoid this interpretive issue, here we focused on a distortion along a single dimension: letter rotation. Importantly, the most influential neural model of word recognition makes a strong prediction in the context of visual word processing: "letter detectors should be disrupted by rotation $\left(>40^{\circ}\right)$ " (Local Combination Detectors [LCD] model: Dehaene, Cohen, Sigman, \& Vinckier, 2005, p. 340). Similarly, Cohen, Dehaene, Vinckier, Jobert, and Montavont (2008, p. 364) stated: "the ventral visual word form system, alone, is limited in its invariance" and would be hindered by rotations above $45^{\circ}$. Consistent with this prediction, a number of singlepresentation experiments have reported shorter response times 
to isolated letters and letter strings when presented horizontally than when rotated $45^{\circ}$ or $90^{\circ}$ (Risko, Medimorec, Chisholm, \& Kingstone, 2014; see Kim \& Straková, 2012, for electrophysiological evidence; see Blythe, Juhasz, Tbaily, Rayner, \& Liversedge, 2019, for evidence during sentence reading; see also Jolicoeur \& Landau, 1984; Jolicoeur, Snow, \& Murray, 1987, for early research with isolated letters). These effects are greater for letter strings than for isolated letters - this has been interpreted in terms of the small time needed to encode a simple pattern (e.g., a letter; see Risko et al., 2014, for discussion).

Critically, this prediction from the LCD model has recently been put into question in several experiments that tapped the initial moments of processing using Forster and Davis' (1984) masked priming procedure (see Grainger, 2008, for a review of the advantages of masked priming over single-presentation techniques). In a lexical decision experiment, Perea, Marcet, and Fernández-López (2018a) found that the magnitude of masked repetition priming (e.g., social-SOCIAL vs. energySOCIAL) and masked transposed-letter priming (e.g., soicalSOCIAL vs. soaral-SOCIAL) was sizeable and similar for words in marquee format (i.e., a vertical format with horizontal letters; 40-ms identity priming effect; 19-ms transposedletter priming effect) and for words rotated $90^{\circ}$ (37-ms identity priming effect; 20-ms transposed-letter priming effect). Clearly, if letter detectors had been disrupted by rotations above $40^{\circ}$ in the first moments of word processing, one would have expected a sizeable reduction of masked repetition/ transposed-letter priming effects for $90^{\circ}$ rotated words. Furthermore, Yang and Lupker (2019) provided an even more extreme demonstration. They conducted two masked priming lexical decision experiments in which the words were presented horizontally or rotated $90^{\circ}$ or $180^{\circ}$. While, unsurprisingly, overall latencies were longer for rotated stimuli (i.e., $180^{\circ}>$ $90^{\circ}>0^{\circ}$ ), the size of the transposed-letter priming effect was similar in the three formats $\left(33,29\right.$, and $35 \mathrm{~ms}$ for $0^{\circ}, 90^{\circ}$, and $180^{\circ}$ words, respectively). Taken together, these two series of experiments suggest that, in the first moments of processing, letter detectors do not seem to be severely hindered by rotation. However, there are two potential limitations in these experiments. First, one might argue that top-down lexical processes might have contributed to cancelling out the effect of rotation angle (i.e., prime-target integration can be enhanced when the stimuli tap onto lexical representations, over-riding physical features (e.g., altar-ALTAR = ALTAR-ALTAR); see Vergara-Martínez, Gomez, Jiménez, \& Perea, 2015, for electrophysiological evidence). Second, participants could have spontaneously used strategies when processing the rotated stimuli to offload cognitive work such as tilting their heads (see Risko et al., 2014, for discussion).

In the present set of experiments, we examined the effects of letter rotation in the first moments of letter processing while avoiding lexical or strategic processes. To do that, we combined a letter match task (i.e., a task that does not involve lexical processing) with a masked priming procedure (see Kinoshita \& Kaplan, 2008; Kinoshita, Robidoux, Mills, \& Norris, 2013). Importantly, both probe and target letters always in different case - were presented in the canonical horizontal format (i.e., only the masked prime was rotated). In this manner, participants could not realize that the prime stimuli were rotated, thus minimizing any potential conscious strategies. The setup of a given trial was the following. A probe letter was presented above a pattern mask for $750 \mathrm{~ms}$. The prime stimulus replaced the mask for $50 \mathrm{~ms}$, which was in turn replaced by the target letter. To effectively mask the rotated primes, the size of both mask and target was greater than the size of the prime (see Fig. 1, for illustration of the technique). The key comparison was between identity primes versus unrelated primes (e.g., probe $\underline{\mathrm{r}}$; prime $\underline{\mathrm{R}}$; target $\underline{\mathrm{R}}$ vs. probe $\underline{\mathrm{r}}$; prime $\underline{\mathrm{T}}$; target $\underline{\mathrm{R}}$ ).

As indicated earlier, previous parametric manipulations of rotation angle with isolated letters have shown a processing cost. In a letter-identification task in which letters were presented briefly and masked, Jolicoeur and Landau (1984) found a higher percent of errors as a function of rotation angle ([0 $0^{\circ}$ vs. $30^{\circ}$ vs. $60^{\circ}$ vs. $\left.90^{\circ}\right]$ Experiment 1: 21.9 vs. 40.6 vs. 57.3 vs. $52.1 \%$, respectively; Experiment 2: 25.0 vs. 25.0 vs. 43.8 vs. $50.0 \%$, respectively). More recently, in a naming task in which the letters were presented at several orientations, Risko et al. (2014) found that response times were modulated by rotation angle ([0 $0^{\circ}$ vs. $45^{\circ}$ vs. $\left.90^{\circ}\right]$ Experiment $1: 823$ vs. 831 vs. 870 ms, respectively; Experiment 2: 963 vs. 1,045 vs. $1,068 \mathrm{~ms}$, respectively). However, none of these experiments can disentangle whether the effect of rotation angle occurred in the initial moments of processing or whether the processing cost occurred later in processing. This is the reason why we opted for a masked priming paradigm with isolated letters: if rotation slows down the initial stages of letter processing, the size of masked identity priming should be reduced for rotated primes beyond $40-45^{\circ}$.

In each of the three experiments reported here, the primes were presented with one of two rotation angles, the difference always being $45^{\circ}$. In Experiment 1, we compared $0^{\circ}$ versus $45^{\circ}$ rotation angles. In Experiment 2, we compared $22.5^{\circ}$ versus $67.5^{\circ}$ rotation angles. In Experiment 3, we compared $45^{\circ}$ versus $90^{\circ}$ rotation angles. If letter detectors are hindered by rotations above $40^{\circ}$ or $45^{\circ}$, as can be inferred from the LCD architecture (Dehaene et al., 2005), masked identity priming should be substantially reduced when the rotation angle of the prime is $45^{\circ}$ or above. That is, one would expect an interaction between rotation and identity priming in Experiments 1 and 2, and a null effect of priming in Experiment 3. Alternatively, if letter detectors are resilient to rotation in the initial moments of processing, as claimed by Perea et al. (2018a, b) and Yang and Lupker (2019), masked identity priming should be approximately similar in magnitude in the two rotation angles in the three experiments. 


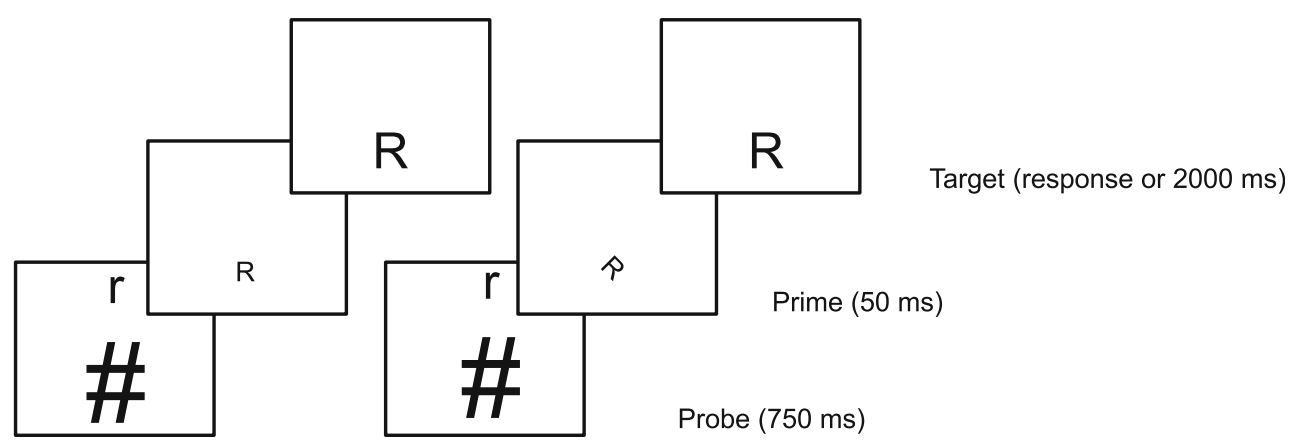

Fig. 1 Schematic representation of a "same" trial and a "different" trial with a masked priming letter match task with a $45^{\circ}$ prime rotation

\section{Method}

\section{Participants}

The sample was composed of 60 students from the University of Valencia with no hearing or reading disorders (20 in each experiment). All of them had normal or corrected-to-normal vision and signed a consent form before starting the experiment. For each experiment, the number of stimuli in each condition (identity condition, unrelated condition) for both "same" and "different" trials was 128 (i.e., $20 * 128=2,560$ observations).

\section{Materials}

We employed eight consonants (B, C, F, G, R, S, T, and V). Probe and targets were always in different case (e.g., r-R, s-T) and primes were presented in the same case as the targets note that Kinoshita and Kaplan (2008) found that identity priming occurred to a similar degree for visually similar pairs (e.g., t-T) and for visually dissimilar pairs (e.g., r-R). For "same" trials, the prime could be the same letter as the target (identity condition) or a different letter (unrelated condition) (e.g., [probe-prime-target] r-R-R vs. r-T-R). For "different" trials, the prime could be the same as the probe or not (e.g., f-F-R vs. f-T-R) - this way, the probe-prime relationship was not predictive of the responses (see Fig. 1, for a scheme of a trial). The rotation angles for the primes were: $0^{\circ}$ versus $45^{\circ}$ (Experiment 1 ), $22.5^{\circ}$ versus $67.5^{\circ}$ (Experiment 2), and $45^{\circ}$ versus $90^{\circ}$ (Experiment 3$)^{1}$

\footnotetext{
${ }^{1}$ As a reviewer pointed out, when rotated $90^{\circ}$, a prime consisting of the lowercase letter $\mathrm{b}$ would also correspond to a $-90^{\circ}$ rotated letter $\mathrm{q}$. This affected a total of eight trials/condition (6.25\%) of "same" responses in the $45^{\circ}$ and $90^{\circ}$ rotations. As this could have potentially reduced the overall masked identity priming effect in Experiment 3 (i.e., the experiment with the $45^{\circ}$ and $90^{\circ}$ rotation angles), we conducted the statistical analyses excluding these trials. The findings were essentially the same as those with the original analyses.
}

\section{Procedure}

The experiments were conducted individually or in groups of up to three participants in a quiet room. DMDX software on Windows computer (Forster \& Forster, 2003) was used to present the stimuli and collect the responses. Participants were told that, on each trial, they would see a sequence of two letters, and they had to decide - as quickly and accurately as possible - whether these two letters were the same or not. On each trial, a probe letter was presented above a pattern mask (\#) on the CRT screen for $750 \mathrm{~ms}$. Then, the probe disappeared and the prime replaced the mask for $50 \mathrm{~ms}$ and, subsequently, the target letter replaced the prime. To effectively mask the primes, the mask was presented in 48-pt Arial font, the prime was presented in 12-pt Arial font, and probes and targets were presented in 22-pt Arial font. The target remained on the screen until the response - there was a 2-s deadline. There were 16 practice trials before then 512 experimental trials in each experiment. The session took approximately $18-22 \mathrm{~min}$.

\section{Results}

As is usual with this procedure, we focused on "same" trials (i.e., trials in which the probe and the target were the same). In the response time (RT) analyses, we excluded the incorrect responses and the correct responses briefer than $250 \mathrm{~ms}$. The mean RTs and error rates in each condition are displayed in Table 1. For the inferential analyses, we employed the lme 4 (Bates, Maechler, Bolker, \& Walker, 2015) and lmerTest packages (Kuznetsova, Brockhoff, \& Christensen, 2016) in R (R Core Team, 2019). In each experiment, the two fixed factors (prime-target relationship [identity, unrelated] and rotation angle) were zero-centered. For the latency data, we employed generalized mixed-models analyses with the Gamma function because they do not require a non-linear transformation of RTs - note that non-linear transformations might (in some scenarios) alter the nature of the interaction between factors (see Yang \& Lupker, 2019, for discussion). Nonetheless, inverse 
Table 1. Mean response times (in $\mathrm{ms}$ ) and percentage of errors (in parentheses) in each of the conditions in the experiment

\begin{tabular}{|c|c|c|c|}
\hline \multirow[t]{2}{*}{ Prime rotation } & \multicolumn{3}{|c|}{ Prime-target relationship } \\
\hline & Identity & Unrelated & Unrelated - identity \\
\hline \multicolumn{4}{|l|}{ Experiment 1} \\
\hline \multicolumn{4}{|l|}{ "Same" trials } \\
\hline $0^{\circ}$ & $497(3.6)$ & $518(7.1)$ & $21(3.5)$ \\
\hline $45^{\circ}$ & $506(5.1)$ & $522(6.6)$ & $16(1.5)$ \\
\hline \multicolumn{4}{|c|}{ "Different" trials } \\
\hline $0^{\circ}$ & $565(3.7)$ & $563(5.1)$ & $-2(1.4)$ \\
\hline $45^{\circ}$ & $558(4.2)$ & $553(3.4)$ & $-5(-0.8)$ \\
\hline \multicolumn{4}{|l|}{ Experiment 2} \\
\hline \multicolumn{4}{|l|}{ "Same" trials } \\
\hline $22.5^{\circ}$ & $532(4.2)$ & $544(5.2)$ & $12(1.0)$ \\
\hline $66.5^{\circ}$ & $530(4.1)$ & $542(3.8)$ & $12(-0.3)$ \\
\hline \multicolumn{4}{|c|}{ "Different" trials } \\
\hline $22.5^{\circ}$ & $597(3.2)$ & $595(3.7)$ & $-2(0.5)$ \\
\hline $66.5^{\circ}$ & $592(3.2)$ & $591(3.0)$ & $-1(-0.2)$ \\
\hline \multicolumn{4}{|l|}{ Experiment 3} \\
\hline \multicolumn{4}{|l|}{ "Same" trials } \\
\hline $45^{\circ}$ & $483(4.3)$ & $498(5.3)$ & $15(1.0)$ \\
\hline $90^{\circ}$ & $479(4.3)$ & $493(4.3)$ & $14(0.0)$ \\
\hline \multicolumn{4}{|c|}{ "Different" trials } \\
\hline $45^{\circ}$ & $546(3.8)$ & $537(2.9)$ & $-9(-0.9)$ \\
\hline $90^{\circ}$ & $546(2.0)$ & $551(3.7)$ & $5(1.7)$ \\
\hline
\end{tabular}

transformations (i.e., -1,000/RT) with linear mixed-effects models and analyses of variance on the participants' and items' means produced the same pattern of findings as that reported here. In the analyses of accuracy (i.e., categorical responses: $1=$ correct; $0=$ error), we employed generalized mixed-models analyses with the binomial function. For each test, we chose the most complex random effect structure that converged. The specific models are indicated in Appendix $1 .^{2}$

\section{Experiment $1\left(0^{\circ}\right.$ vs. $\left.45^{\circ}\right)$}

Latency data We found faster responses in the identity condition than in the unrelated condition, $b=17.215, S E=4.794, z$ $=3.591, p<.001$. In addition, responses were faster when the prime was in horizontal format than in the $45^{\circ}$ format, $b=$ 9.358, $S E=3.866, z=-2.421, p=.015$. The interaction between the two factors did not approach significance $(z<1, p>$ $.45)$.

\footnotetext{
${ }^{2}$ For completeness, we also examined the effects for "different" trials - in these trials, probe and targets were always unrelated (e.g., f-F-R vs. f-T-R) and the relationship was between the probe and the prime. None of the identity priming effects in Experiments 1, 2, or 3 was significant (see Carreiras, Perea, \& Abu Mallouh, 2012, for a similar pattern with a letter match task; but see Kinoshita, Robidoux, Mills, \& Norris, 2013, for an inhibitory effect).
}

Accuracy data The data showed a small numerical advantage of the identity priming condition, but neither the main effects nor their interaction was significant (priming: $b=-0.381, S E=$ $0.204, z=-1.869, p=.062$; rotation: $b=0.387, S E=0.201, z=$ $1.93, p=.054$; interaction: $b=-0.480, S E=0.257, z=-1.869$, $p=.062)$

\section{Experiment $2\left(22.5^{\circ}\right.$ vs. $\left.67.5^{\circ}\right)$}

Latency data The results showed an advantage of the identity over the unrelated condition, $b=14.675, S E=3.678, z=$ $3.990, p<.001$, whereas the other factors did not approach significance (both $\mathrm{zs}<1, p \mathrm{~s}>.61$ ).

Accuracy data None of the effects approached significance (all $z \mathrm{~s}<1.4$, all $p \mathrm{~s}>.15$ ).

\section{Experiment $3\left(45^{\circ}\right.$ vs. $\left.90^{\circ}\right)$}

Latency data Response times were faster in the identity condition than in the unrelated condition, $b=15.785, S E=4.707$, $z=3.354, p<.001$. The other factors did not approach significance (both $z \mathrm{~s}<1$, both $p \mathrm{~s}>.45$ ).

Accuracy data None of the effects approached significance (all $z \mathrm{~s}<1.6$, all $p \mathrm{~s}>.11$ ).

\section{Discussion}

While there is some agreement that rotated letters are identified more slowly than their canonical upright counterparts (see Risko et al., 2014, for review), it is unclear whether this effect has its locus in the initial stages of processing. In the current set of experiments, we examined whether letter detectors are disrupted by rotation angle up to $90^{\circ}$ during the first stages of processing. To that end, we manipulated the rotation angle of letter primes in three masked identity priming letter match experiments. We found a sizeable masked identity priming effect that was comparable in magnitude regardless of the rotation angle of the prime (Experiment 1: $0^{\circ}$ vs. $45^{\circ}$; Experiment 2: $22.5^{\circ}$ vs. $67.5^{\circ}$; Experiment $3: 45^{\circ}$ vs. $90^{\circ}$ ). Critically, this pattern occurred in a scenario in which participants could not anticipate that some of the stimuli were rotated: all probe and target stimuli were presented in the standard upright orientation. While identity priming was not affected by letter rotation, we did find a small overall cost of letter rotation in Experiment $1\left(0^{\circ}\right.$ vs. $\left.45^{\circ}\right)$. However, this effect must be interpreted with some caution, as it may reflect an effect of congruency between the rotation angle of the prime and the rotation angle of the probe/target - consistent with this interpretation, this difference was absent in Experiments 2 and 3. 
The pattern of priming effects in these letter-match experiments is consistent with the robust masked identity and form priming effects in the lexical decision task reported by Perea et al. (2018a, b) and Yang and Lupker (2019) with $90^{\circ}$ rotated words. The most parsimonious explanation of these findings is that, in the early stages of letter and word processing, letter detectors are resilient to rotation angles of $90^{\circ}$ or below. Thus, the processing cost that occurs when identifying isolated rotated letters (Risko et al., 2014) or rotated words (see Yu, Gerold, Park, \& Legge, 2010) would not be due to a disruption during the initial stages of processing but instead it would occur at a later processing stage (see Forster, 1998, for a similar argument concerning mixed-case stimuli).

Thus, the current experiments, together with the Perea et al. (2018a, b) and Yang and Lupker (2019) experiments, pose some problems for the LCD model of visual word recognition (Dehaene et al., 2005). This model establishes clear predictions regarding the tolerance of the visual system to different types of stimulus degradation during word reading. Specifically, in the context of fast word recognition, letter detectors should be disrupted by rotation angles larger than $45^{\circ}$. But why did Dehaene et al. (2005) make the claim that a rotation of around $40-45^{\circ}$ would mark the limit of resilience in letter detectors? The key basis of their claim was based on an influential experiment conducted by Logothetis and Pauls (1995) with monkeys. These primates had been trained with novel wire-like and spheroidal 3D objects that were always presented with a specific viewpoint. In a subsequent phase, the monkeys were presented with these objects, but in various angles. The monkeys' ability in identifying these objects decreased dramatically in rotation angles of around $40^{\circ}$ or $45^{\circ}$. However, these findings, which were obtained after presenting various $3 \mathrm{D}$ objects with a single viewpoint to a non-human species, may not be generalizable to the letter detectors of the reading system in humans. Leaving aside that letters are $2 \mathrm{D}$ objects, compared to the artificial 3D objects used in the Logothetis and Pauls (1995) experiment, the tuning of letter detectors (i.e., the groups of neurons that respond to a given letter in humans) is fed by experience with multiple different printed versions of the same letter (e.g., $\mathrm{A}, \mathrm{A}, A)$. In order to rapidly recognize each of a word's constituent letters, the visual system encodes basic-level differentiating features while over-riding subordinatelevel differences (e.g., font, size, etc.). As a result, letter detectors may be more resistant to variations in the visual form of the letters than the groups of neurons that respond to a trained $3 \mathrm{D}$ object in a single viewpoint in monkeys (see Hannagan et al., 2012, for discussion).

To sum up, the present masked priming experiments demonstrated that the visual form processing system is resilient to rotation angles of $90^{\circ}$ or below in the initial moments of letter processing. This pattern supports the claims that, as a result of extensive experience, the human brain has developed a perceptual expertise for processing letters and words independently of the form (i.e., the abstract unit "e" would be activated with the visual input e, e, or (D ). As Koriat and Normal (1985) anticipated, the extensive practice in letter/word identification results in the "establishment of a broadly tuned memory representation that allows direct stimulus recognition over a relatively wide range of stimulus orientations" (p. 438). What the current set of experiments revealed is that the "internal rectifying operations" to reconstruct a canonical version of a rotated letter seem to occur very rapidly. Further experiments should examine in depth the time course of letter rotation (e.g., using electrophysiological measures) not only with isolated letters but also with words composed of rotated letters.

Open practice statement All stimuli, data, and scripts are available at https://osf.io/qru9k/.

Funding Information This work was supported by the Spanish Ministry of Science, Innovation, and Universities [PRE2018-083922, PSI201786210-P].

\section{Appendix}

\section{Linear Mixed-Effects Models in each of the Experiments}

\section{Experiment 1}

RT data: RT_GLME = glmer $(\mathrm{rt} \sim$ prime*rotation $+(1+$ prime $*$ rotation $\mid$ subject $)+(1+$ prime $\mid$ item $)$, data $=$ rotation $1 \mathrm{tr}$, family = Gamma(link="identity"))

Accuracy data: accuracy_glme = glmer(accuracy $\sim$ prime $*$ rotation $+(1+$ prime $\mid$ subject $)+(1+$ prime $\mid$ item $)$, data $=$ rotation 1 , family $=$ binomial $)$

\section{Experiment 2}

RT data $:$ RT_LME $=$ glmer $(\mathrm{rt} \sim$ prime*rotation $+(1 \mid$ subject $)+$ (1 1 item), data $=$ rotation2tr, family = Gamma(link="identity"))

Accuracy data: accuracy_glme $=\operatorname{glmer}($ accuracy $\sim$ prime*rotationc+ $(1+$ prime $\mid$ subject $)+(1 \mid$ item $)$, data $=$ rotation2, family $=$ binomial)

\section{Experiment 3}

RT data: RT_GLME= glmer $(\mathrm{rt} \sim$ prime*rotation $+(1+$ prime*rotation $\mid$ subject $)+(1+$ prime+rotation $\mid$ item $)$, data $=$ rotation 3 tr, family $=$ Gamma(link="identity")) 
Accuracy data: accuracy_glme = glmer(accuracy $~$ prime $*$ rotationc $+(1+$ prime $\mid$ subject $)+(1+$ prime $\mid$ item $)$, data $=$ rotation3, family = binomial)

\section{References}

Bates, D., Maechler, M., Bolker, B., \& Walker, S. (2015). Fitting linear mixed-effects models using lme4. Journal of Statistical Software, 67, 1-48. 10.18637/jss.v067.i01

Blythe, H. I., Juhasz, B. J., Tbaily, L. W., Rayner, K., \& Liversedge, S. P. (2019). Reading sentences of words with rotated letters: An eye movement study. Quarterly Journal of Experimental Psychology. https://doi.org/10.1177/1747021818810381

Carreiras, M., Perea, M., \& Mallouh, R. A. (2012). Priming of abstract letter representations may be universal: The case of Arabic. Psychonomic Bulletin \& Review, 19, 685-690. https://doi.org/10. 3758/s13423-012-0260-8

Cohen, L., Dehaene, S., Vinckier, F., Jobert, A., \& Montavont, A. (2008). Reading normal and degraded words: Contribution of the dorsal and ventral visual pathways. Neuroimage, 40, 353-366. https://doi.org/ 10.1016/j.neuroimage.2007.11.036

Dehaene, S., Cohen, L., Sigman, M., \& Vinckier, F. (2005). The neural code for written words: A proposal. Trends in Cognitive Sciences, 9, 335-341. https://doi.org/10.1016/j.tics.2005.05.004

Forster, K. I. (1998). The pros and cons of masked priming. Journal of Psycholinguistic Research, 27, 203-233. https://doi.org/10.1023/A: 1023202116609.

Forster, K. I., \& Davis, C. (1984). Repetition priming and frequency attenuation in lexical access. Journal of Experimental Psychology: Learning, Memory, and Cognition, 10, 680-698. https://doi.org/10. 1037/0278-7393.10.4.680

Forster, K. I., \& Forster, J. C. (2003). DMDX: A Windows display program with millisecond accuracy. Behavior Research Methods, Instruments, \& Computers, 35, 116-124. https://doi.org/10.3758/ BF03195503

Grainger, J. (2008). Cracking the orthographic code: An introduction. Language and Cognitive Processes, 23, 1-35. https://doi.org/10. 1080/01690960701578013

Grainger, J. \& Dufau, S. (2012). The front-end of visual word recognition. In J. S. Adelman (Ed.), Visual Word Recognition Vol. 1: Models and Methods, Orthography and Phonology (pp. 159-184). Hove, UK: Psychology Press.

Hannagan, T., Ktori, M., Chanceaux, M., \& Grainger, J. (2012). Deciphering CAPTCHAs: What a Turing test reveals about human cognition. PloS ONE, 7, e32121. https://doi.org/10.1371/journal. pone. 0032121

Jolicoeur, P., \& Landau, M. J. (1984). Effects of orientation on the identification of simple visual patterns. Canadian Journal of Psychology, 38, 80-93. https://doi.org/10.1016/10.1037/h0080782

Jolicoeur, P., Snow, D., \& Murray, J. (1987). The time to identify disoriented letters: Effects of practice and font. Canadian Journal of Psychology, 41, 303-316. https://doi.org/10.1037/h0084159
Kim, A. E., \& Straková, J. (2012). Concurrent effects of lexical status and letter-rotation during early stage visual word recognition: Evidence from ERPs. Brain Research, 1468, 52-62. https://doi.org/10.1016/j. brainres.2012.04.008

Kinoshita, S., \& Kaplan, L. (2008). Priming of abstract letter identities in the letter match task. Quarterly Journal of Experimental Psychology, 61, 1873-1885. https://doi.org/10.1080/ 17470210701781114

Kinoshita, S., Robidoux, S., Mills, L., \& Norris, D. (2013). Visual similarity effects on masked priming. Memory \& Cognition, 42, 821833. https://doi.org/10.3758/s13421-013-0388-4

Koriat, A., \& Norman, J. (1985). Mental rotation and visual familiarity. Perception \& Psychophysics, 37, 429-439. https://doi.org/10.3758/ BF03202874

Kuznetsova, A., Brockhoff, P. B., \& Christensen, R. H. B. (2016). ImerTest: Tests for random and fixed effects for linear mixed effect models (Imer objects of lme4 package). R package Version 2.0-33. Retrieved from https://cran.r-project.org/web/packages/lmerTest/

Logothetis, N. K., \& Pauls, J. (1995). Psychophysical and physiological evidence for viewer-centered object representations in the primate. Cerebral Cortex, 5, 270-288. https://doi.org/10.1093/cercor/5.3. 270

Perea, M., Marcet, A., \& Fernández-López, M. (2018a). Does letter rotation slow down orthographic processing in word recognition? Psychonomic Bulletin \& Review, 25, 2295-2300. https://doi.org/ 10.3758/s13423-017-1428-z

Perea, M., Marcet, A., Uixera, B., \& Vergara-Martínez, M. (2018b). Eye movements when reading sentences with handwritten words. Quarterly Journal of Experimental Psychology, 71, 20-27. https:// doi.org/10.1080/17470218.2016.1237531

R Core Team (2019). R: A language and environment for statistical computing. Vienna: R Foundation for Statistical Computing. Retrieved from https://www.r-project.org/

Risko, E. F., Medimorec, S., Chisholm, J., \& Kingstone, A. (2014). Rotating with rotated text: A natural behavior approach to investigating cognitive offloading. Cognitive Science, 38, 537-564. https:// doi.org/10.1111/cogs.12087

Vergara-Martínez, M., Gómez, P., Jiménez, M., \& Perea, M. (2015). Lexical enhancement during prime-target integration: ERP evidence from matched-case identity priming. Cognitive, Affective, \& Behavioral Neuroscience, 15, 492-504. https://doi.org/10.3758/ s13415-014-0330-7

Yang, H., \& Lupker, S. J. (2019). Does letter rotation decrease transposed letter priming effects? Journal of Experimental Psychology: Learning, Memory, and Cognition. https://doi.org/10.1037/ $\mathrm{x} \operatorname{lm} 0000697$

Yu, D., Gerold, D., Park, H., \& Legge, G. E. (2010). Reading horizontal and vertical English text. Journal of Vision, 8, 629-629. https://doi. org/10.1167/8.6.629

Publisher's note Springer Nature remains neutral with regard to jurisdictional claims in published maps and institutional affiliations. 